\title{
El perfil profesional y su relación con el desempeño laboral del egresado del Programa de Estudios de Administración de Empresas de la Universidad Nacional Toribio Rodríguez de Mendoza de Amazonas entre los años 2014 -2017.
}

\section{The professional profile and its relationship with the labor performance of the graduate of the Program Of Studies Of Administration of Companies of the National University Toribio Rodríguez De Mendoza De Amazonas between the years 2014 - 2017.}

\author{
River Chávez Santos ${ }^{1}$, Migdonio Epiquién Chancahuana ${ }^{2}$
}

\section{RESUMEN}

Objetivo: evaluar la relación que hay entre el perfil profesional con el desempeño laboral del egresado del Programa de Estudios de Administración de Empresas de la Universidad Nacional "Toribio Rodríguez de Mendoza" de Amazonas durante los años 2014 - 2017. Metodológicamente el trabajo se aborda desde una perspectiva de la investigación del nivel descriptivo correlacional con un método hipotético deductivo y un diseño no experimental de corte transversal. La población estuvo conformada por 320 egresados de la Escuela Profesional de Administración de Empresas, y la muestra probabilística fue de 175 egresados. La técnica empleada fue la encuesta con el instrumento de recojo de datos el cuestionario de tipo Likert. Resultados: El 32\% de los egresados demuestran tener un perfil profesional poco satisfactorio frente a un desempeño laboral de nivel regular, el $26 \%$ de los egresados demuestran tener un perfil profesional poco satisfactorio frente a un desempeño laboral de nivel deficiente, el $12 \%$, demuestran tener un perfil profesional poco insatisfactorio frente a un desempeño laboral de nivel deficiente, el 10\% de los egresados demuestran tener un perfil profesional poco satisfactorio frente a un desempeño laboral de nivel regular, el 8\% de los egresados demuestran tener un perfil profesional insatisfactorio frente a un desempeño laboral de nivel regular. Conclusiones: 1. En cuanto a la relación: el $32 \%$ manifiestan tener un perfil profesional poco satisfactorio frente a un $26 \%$ de desempeño regular, y la mayoría de egresados manifiestan en una relación poco satisfactoria frente a un desempeño laboral deficiente. 2. Un $24 \%$ de egresados manifiestan competencias genéricas instrumentales con desempeños laborales deficientes, y la mayoría manifiestan una relación insatisfactoria y deficiente. 3. El 30\% de los egresados demuestran tener competencias genéricas interpersonales poco satisfactorias frente a un desempeño laboral de nivel regular, el 20\% de los egresados demuestran tener competencias genéricas interpersonales satisfactorias frente a un desempeño laboral de nivel regular, y la mayoría de egresados manifiestan en una relación de regular a deficiente. 4. El 36\% de los egresados demuestran tener competencias genéricas sistémicas poco satisfactorias frente a un desempeño laboral de nivel regular, y los demás porcentajes en una relación poco satisfactorio y deficiente.

Palabras clave: perfil profesional, desempeño laboral.

\section{ABSTRACT}

Objective: to evaluate the relationship between the professional profile and the work performance of the graduate of the Business Administration Studies Program of the National University "Toribio Rodríguez de Mendoza" of Amazonas during the years 2014 - 2017. Methodologically the work is approached from a perspective of the investigation of the descriptive correlational level with a hypothetical deductive method and a non-experimental cross-sectional design. The population consisted of 320 graduates of the Professional School of Business Administration, and the probabilistic sample was 175 graduates. The technique used was the survey with the instrument of data collection, the Likert type questionnaire. Results: $32 \%$ of the graduates show an unsatisfactory professional profile compared to a regular work performance, $26 \%$ of the graduates show an unsatisfactory professional profile compared to a poor level of work performance, $12 \%$, they show that they have a low 
unsatisfactory professional profile in comparison to a poor level of work performance, $10 \%$ of graduates show an unsatisfactory professional profile compared to a regular work performance, $8 \%$ of graduates show an unsatisfactory professional profile in front of a work performance of regular level. Conclusions: 1 . Regarding the relationship: $32 \%$ said they had an unsatisfactory professional profile compared to $26 \%$ of regular performance, and the majority of graduates expressed an unsatisfactory relationship with poor work performance. 2. 24\% of graduates manifest instrumental generic competencies with deficient work performance, and most of them show an unsatisfactory and deficient relationship. $3.30 \%$ of the graduates show unsatisfactory interpersonal generic competences compared to a regular level of work performance, $20 \%$ of the graduates demonstrate having satisfactory interpersonal generic competences in front of a regular work performance, and the majority of graduates manifest in a relationship of regular to deficient. $4.36 \%$ of the graduates show unsatisfactory systemic generic competences in comparison with a regular level of work performance, and the other percentages in an unsatisfactory and deficient relationship.

Keywords: professional profile, job performance.

${ }^{\mathrm{I}} \mathrm{M}$ 


\section{INTRODUCCIÓN}

La Universidad Nacional Toribio Rodríguez de Mendoza, se está convirtiendo en un escenario competitivo, por lo cual es necesario que establezca y formule estrategias que conduzcan a superar retos para el acceso a estudios de formación profesional de tal manera que los egresados incursionen en igualdad de condiciones hacia los campos de la competitividad en los trabajos que les corresponda desempeñarse, los mismos que deben estar ligados a la ciencia, la tecnología y la investigación. En ese sentido, para emprender las actividades investigativas, se ha partido del problema, formulando la pregunta ¿Cuál es la relación entre el perfil profesional con el desempeño laboral de los egresados de la Escuela Profesional de Administración de Empresas de la Universidad Nacional "Toribio Rodríguez de Mendoza" entre los años 2014 - 2017?. El problema que motivó la investigación es el desempeño laboral, que en cualquiera de los campos exige la participación de profesionales y trabajadores competentes, formados en los ámbitos de las universidades y entidades de educación superior imbuidos en el enfoque de las competencias, que deben dar como resultados a profesionales con perfiles de calidad para desenvolverse con capacidad a cualquier contexto de trabajo, con eficiencia, con idoneidad para solucionar problemas, diseñar y ejecutar proyectos, promover el desarrollo del lugar donde se encuentran laborando. El estudio nos llevó a realizar una descripción de la relación entre las variables: perfil profesional como variable 1 y el desempeño laboral como variable 2., de los egresados de la Escuela Profesional de Administración de empresas. El estudio se sustenta en teorías del enfoque por competencias, que es considerado como la capacidad de conocer, hacer y actuar, que están presentes en el diario vivir del profesional a través de las competencias genéricas instrumentales, interpersonales y sistémicas.

La finalidad de la presente investigación es conocer el tipo de relación que hay entre el perfil profesional del que egresa de la Escuela Profesional de Administración de Empresas con la calidad de desempeño en el trabajo donde se encuentre, para cerciorarse de las debilidades y fortalezas en formación de profesionales a través de enseñanza aprendizaje, en la mencionada Escuela Profesional.

\section{MATERIAL Y MÉTODOS}

Corresponde a la investigación básica del nivel descriptivo correlacional, con un diseño de estudio no experimental de corte transversal, para establecer la relación existente entre la variable 1 y la variable 2 . La Técnica empleada fue la encuesta con su instrumento de obtención de los datos, el cuestionario con la escala Likert, el mismo que fue validado por tres expertos, fue aplicado a una muestra de 175 egresados de la Escuela Profesional de Administración de Empresas.

\section{RESULTADOS}

Después del procesamiento de los datos obtenidos y sometidos a los estadísticos correspondientes, se obtuvo los siguientes resultados:

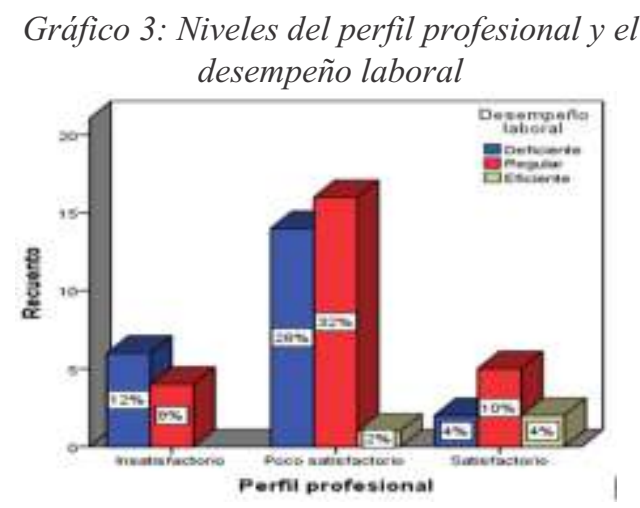

Fuente: Chávez S. River y Epiquién Ch. Migdonio

En la figura se establece que el $32 \%$ de los egresados demuestran tener un perfil profesional poco satisfactorio frente a un desempeño laboral de nivel regular, el $26 \%$ de los egresados demuestran tener un perfil profesional poco satisfactorio frente a un desempeño laboral de nivel deficiente, el $12 \%$ de los egresados demuestran tener un perfil profesional poco insatisfactorio frente a un desempeño laboral de nivel deficiente, el $10 \%$ de los egresados demuestran tener un perfil profesional poco satisfactorio frente a un desempeño laboral de nivel regular, el $8 \%$ de los egresados demuestran tener un perfil profesional insatisfactorio frente a un desempeño laboral de nivel regular.

Figura 4: Niveles de competencias genéricas instrumentales $v$ desempeño laboral

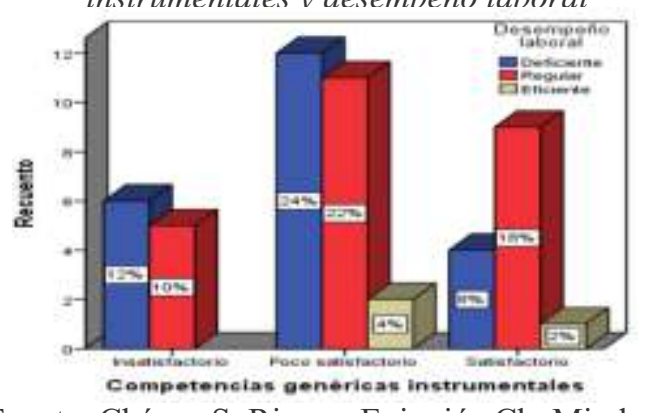

Fuente: Chávez S. River y Epiquién Ch. Migdonio 
En la figura 4 se establece que el $24 \%$ de los egresados demuestran tener competencias genéricas instrumentales poco satisfactorio frente a un desempeño laboral de nivel deficiente, el 22\% de los egresados demuestran tener competencias genéricas instrumentales poco satisfactorio frente a un desempeño laboral de nivel deficiente, el $16 \%$ de los egresados demuestran tener un perfil profesional satisfactorio frente a un desempeño laboral de nivel regular, el $12 \%$ de los egresados demuestran tener un perfil profesional insatisfactorio frente a un desempeño laboral de nivel deficiente, el 10\% de los egresados demuestran tener un perfil profesional insatisfactorio frente a un desempeño laboral de nivel regular, el $8 \%$ de los egresados demuestran tener un perfil profesional satisfactorio frente a un desempeño laboral de nivel deficiente.

Figura 5. Niveles de las competencias genéricas interpersonales y desempeño laboral

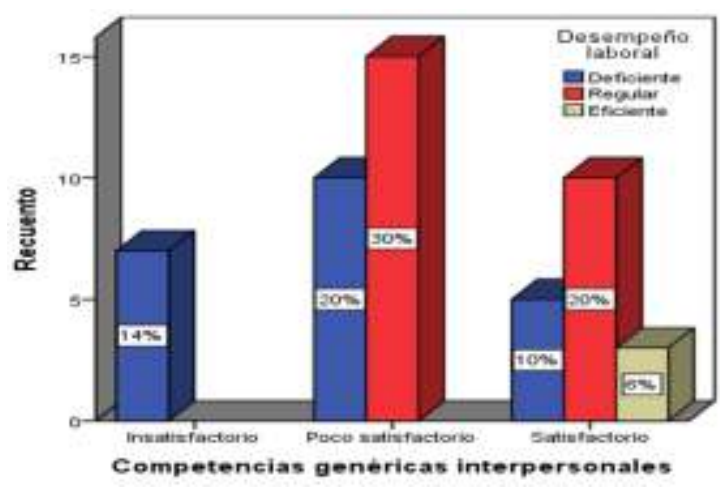

Fuente: Chávez S. River y Epiquién Ch. Migdonio

En la figura 5 se establece que el $30 \%$ de los egresados demuestran tener competencias genéricas interpersonales poco satisfactorio frente a un desempeño laboral de nivel regular, el $20 \%$ de los egresados demuestran tener competencias genéricas interpersonales satisfactorio frente a un desempeño laboral de nivel regular, el $20 \%$ de los egresados demuestran tener un perfil profesional poco satisfactorio frente a un desempeño laboral de nivel deficiente, el 14\% de los egresados demuestran tener un perfil profesional insatisfactorio frente a un desempeño laboral de nivel deficiente, el 10\% de los egresados demuestran tener un perfil profesional satisfactorio frente a un desempeño laboral de nivel deficiente, el $6 \%$ de los egresados demuestran tener un perfil profesional satisfactorio frente a un desempeño laboral de nivel eficiente.
Figura 6. Niveles de las competencias genéricas sistémicas y desempeño laboral.

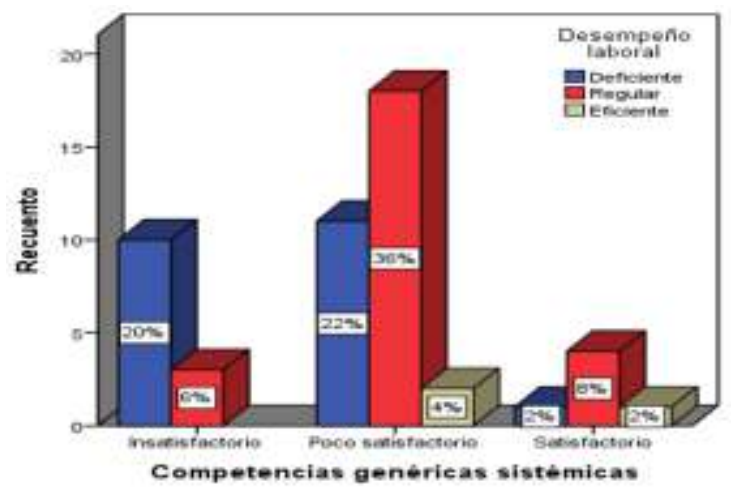

Fuente: Chávez S. River y Epiquién Ch. Migdonio

En la figura 6 se establece que el $36 \%$ de los egresados demuestran tener competencias genéricas sistémicas poco satisfactorio frente a un desempeño laboral de nivel regular, el $22 \%$ de los egresados demuestran tener competencias genéricas sistémica poco satisfactorio frente a un desempeño laboral de nivel deficiente, el $20 \%$ de los egresados demuestran tener un perfil profesional insatisfactorio frente a un desempeño laboral de nivel deficiente, el $8 \%$ de los egresados demuestran tener un perfil profesional satisfactorio frente a un desempeño laboral de nivel regular, el $9 \%$ de los egresados demuestran tener un perfil profesional insatisfactorio frente a un desempeño laboral de nivel regular, el $4 \%$ de los egresados demuestran tener un perfil profesional poco satisfactorio frente a un desempeño laboral de nivel eficiente.

\section{DISCUSIÓN}

Respecto al perfil profesional y el desempeño laboral de los egresados de la Escuela Profesional de Administración de Empresas no hay relación directa ni proporcional. Resultado que guarda relación con Méndez (2015) en su tesis titulada Perfil profesional y la satisfacción de la profesión de la profesión elegida en estudiantes de décimo ciclo de la Facultad de Arquitectura de la Universidad Ricardo Palma, en uno de sus resultados dice que el perfil profesional no se relacionó de manera significativa con la satisfacción con la profesión elegida en estudiantes del décimo ciclo de la facultad de Arquitectura de la Universidad Ricardo Palma.

Referente a la relación entre la competencia genérica instrumental y su relación con el desempeño laboral se deduce que no existe una relación satisfactoria, todo oscila entre desempeños laborales deficientes e insatisfactorios, el $24 \%$ de los egresados demuestran tener competencias genéricas instrumentales poco satisfactorio frente a un desempeño laboral de nivel 
deficiente, Méndez (2015) señala lo contrario en uno de sus resultados en su tesis: "Perfil profesional y la satisfacción de la profesión de la profesión elegida en estudiantes de décimo ciclo de la Facultad de Arquitectura de la Universidad Ricardo Palma, en la que encuentra una relación caracterizada en forma más positiva y satisfactoria entre perfil profesional y la satisfacción de la profesión elegida. Así mismo en cuanto a la relación de las competencias genéricas interpersonales y las competencias genéricas sistémicas con el desempeño laboral se deduce que existe una relación poco satisfactoria frente a un desempeño laboral de un nivel deficiente, en relación de forma contraria y significativa Felipe (2016) con su tesis titulada: "Satisfacción del empleador con relación al desempeño de los egresados de la carrera profesional de Enfermería de la ULADECH Católica señala que este tipo de competencias son favorables en sus trabajos en las instituciones de salud de Chimbote.

\section{CONCLUSIONES}

1. En cuanto a la relación: el $32 \%$ manifiestan tener un perfil profesional poco satisfactorio frente a un 26 $\%$ de desempeño regular, y la mayoría de egresados manifiestan en una relación poco satisfactoria frente a un desempeño laboral deficiente.

2. Un $24 \%$ de egresados manifiestan competencias genéricas instrumentales con desempeños laborales deficientes, y la mayoría manifiestan una relación insatisfactoria y deficiente.

3. El $30 \%$ de los egresados demuestran tener competencias genéricas interpersonales poco satisfactorias frente a un desempeño laboral de nivel regular, el $20 \%$ de los egresados demuestran tener competencias genéricas interpersonales satisfactorias frente a un desempeño laboral de nivel regular, y la mayoría de egresados manifiestan en una relación de regular a deficiente.

4. El 36\% de los egresados demuestran tener competencias genéricas sistémicas poco satisfactorias frente a un desempeño laboral de nivel regular, y los demás porcentajes en una relación poco satisfactorio y deficiente.

\section{SUGERENCIAS}

1. A las autoridades representativas y administrativas de la Universidad Nacional Toribio Rodríguez de Mendoza, para que dispongan de forma efectiva el desarrollo curricular en todas las carreras, de acuerdo al enfoque por competencias para la formación de profesionales competentes que puedan desenvolverse profesionalmente de acuerdo al contexto donde se encuentren, y de manera autónoma eficiente e innovadora. 2. A la Escuela de Administración de Empresas de la Facultad de Ciencias Económicas y Administrativas, que planifiquen, implementen y ejecuten los planes curriculares en base a competencias, para el logro de perfiles profesionales de calidad para que cuando egresen demuestren sus aprendizajes de manera competente durante el desempeño laboral, y en el lugar donde se encuentren, demostrando conocimientos científicos, procedimientos estratégicos y con actuaciones basados en la ética y la moral.

\section{REFERENCIAS BIBLIOGRÁFICAS}

Beneitone (2007) Reflexiones y Perspectivas de la Educación Superior en América Latina. Informe Final - Proyecto Tuning. España. RGM. S.A.

Caballero (2011), Metodología Integral Innovadora para planes y tesis. Lima - Perú. Primera Edición. Empresa Editora El Comercio S.A.

Castillo y Cobrerizo (2010), Evaluación Educativa de Aprendizajes y Competencias, España. Pearson Educación SA.

Flores (2010), Propuesta del Modelo de Seguimiento a E gres ados. Internet/Content/9XGXON98/MP9003 09242(1).jpg

Hawes y Corbolan (2005) Construcción de un Perfil Profesional, Universidad de Talca Chile.

Hernández, Fernández y Baptista (2014), Metodología de la Investigación. México, Bogotá. Sexta Edición. McGraw Hill Education.

Ley N ${ }^{\mathrm{a}} 28044$ Ley General de Educación.

Ley 28740, Ley del SINEACE.

Manual de CONEAU: Estándares de Calidad en la Educación Universitaria.

Ministerio de Educación (2002), Perfil del Egresado, Dirección Nacional de Educación Bilingüe e Interculturalidad, DINEBILima-Perú.

Rivera (2003) El Perfil del Egresado. Punto de partida para la Revisión Curricular, Universidad de Puerto Rico.

Valderra (2013) Pasos para elaborar Proyectos de Investigación Científica, Lima-Perú. Segunda Edición, Editorial San Marcos E.I.R.L, Editor.

Vargas (2009), Perfiles de Competencias demandados por cinco sectores productivos en la ciudad de Tijuana- 
México.

Zepeda y Ortiz (2012) "Modelo para Evaluar la Formación y el Desempeño Docente de Egresados de Instituciones de Educación Normal en México-México.

Araya (2015) "Perfil del egresado de la carrera de fonoaudiología de una universidad en la región Metropolitana-México.

Silva (2016) "Aseguramiento de la calidad del perfil de egreso en las carreras de Pedagogía de la Universidad Adventista de Chile"-Chile.

Felipe (2016) "Satisfacción del empleador con relación al desempeño de los egresados de la carrera profesional de Enfermería de la ULADECH Católica en las instituciones de salud de Chimbote, 2015-Chimbote.

Tineo (2012)"Validación del perfil profesional basado en competencias de docentes de institutos de educación superior tecnológico y su grado de aceptación de profesores, a nivel nacional" - Lima.

Acosta (2015) "Influencia de la competencia docente en el desempeño laboral de los egresados del ciclo básico del centro técnico productivo parroquial Sagrado Corazón de Jesús, San Juan de Miraflores-Lima.

Gómez (2013) Desempeño laboral del Licenciado en Turismo de la Universidad de Oriente, según la opinión del sector empresarial turístico privado de la isla de Margarita, estado Nueva Esparta, Venezuela.

Romero y Urdaneta (2012) Desempeño laboral y calidad de servicio del personal administrativo en la Universidades privadas, Universidad Dr. Carlos Belloso Chacin, Maracaibo, Estado de Zulia. Venezuela. 